Annuaire suisse de politique de développement

2 | 1982

Annuaire Suisse - Tiers Monde 1982

\title{
Le rôle des organisations privées suisses de coopération au développement
}

Die Rolle des Schweizerischen Nicht-Staatlichen Entwicklungsorganisationen

\section{Gilbert Rist}

\section{(2) OpenEdition}

\section{Journals}

Édition électronique

URL : http://journals.openedition.org/aspd/1124

DOI : $10.4000 /$ aspd. 1124

ISSN : 1663-9669

Éditeur

Institut de hautes études internationales et du développement

\section{Édition imprimée}

Date de publication : 31 août 1982

ISSN : 1660-5934

\section{Référence électronique}

Gilbert Rist, "Le rôle des organisations privées suisses de coopération au développement ", Annuaire suisse de politique de développement [En ligne], 2 | 1982, mis en ligne le 21 janvier 2013, consulté le 08 septembre 2020. URL : http://journals.openedition.org/aspd/1124; DOI : https://doi.org/10.4000/ aspd. 1124 


\title{
LE ROLE DES ORGANISATIONS PRIVEES SUISSES DE COOPERATION AU DEVELOPPEMENT
}

\author{
Gilbert Rist
}

\section{DIE ROLLE DER SCHWEIZERISCHEN NICHT-STAATLICHEN ENTWICKLUNGSORGANISATIONEN}

Zusammenfassung : Die öffentlichen Mittel der Entwicklungszusammenarbeit sind in der Schweiz gering $(0,25 \%$ des Bruttosozialproduktes), aber die Gesamtbeiträge an die Dritte Welt sind, im Vergleich zum BSP, die höchsten auf Weltebene. Dies ergibt sich durch die Höhe der privaten Investitionen und Kredite. Ein anderer Teil dieser nichtstaatlichen Zuflüsse ist auch beträchtlich, nämlich die Spenden der entwicklungspolitischen Organisationen. Die etwa hundert Millionen Franken, die 1981 gesammelt wurden, machen 25\% der ganzen öffentlichen Mittel der Entwicklungszusammenarbeit aus. Fünf Gruppen solcher schweizerischer Organisationen sind zu unterscheiden. Erstens, die Missionsbewegungen, die als erste Entwicklungshilfe betrieben, dann Organisationen, die in den Vierzigjahren erschienen sind, und die sich nach dem Wiederaufbau Europas an die Dritte Welt wendeten (HEKS, Swissaid). In den Sechzigerjahren wurden die ersten Entwicklungsorganisationen gegründet (Helvetas, Swisscontact), und später solche, die sich an die schweizerische Offentlichkeit richteten (EvB, Arbeitsgruppe Dritte Welt, CETIM, usw.). Als letzte kamen solche, die einen gerechten Handel mit der Dritte Welt fördern wollten (OS3, Dritte-Welt-Läden). Der "Dienst für technische Zusammenarbeit" wurde 1961 geschaffen. Die verschiedenen Organisationen, die schon länger existierten und deshalb mehr Erfahrung besassen, konnten auch weiterhin die offizielle Linie im Bereich der Entwicklungshilfe beinflussen. Das Gesetz über Entwicklungszusammenarbeit trat 1975 in Kraft. Paragraph 5 des Gesetzes verlangte, dass öffentliche Mittel grundsätzlich an die am meisten benachteiligten Bevölkerungsgruppen gehen müssen. Eine solche Forderung war nur durch den grossen Einfluss der verschiedenen Entwicklungsorganisationen möglich gewesen.

On souligne fréquemment la modestie de I'aide publique suisse au développement $(0,24 \%$ du PNB), et l'on ajoute aussitôt que le total des apports est finalement le plus élevé du monde par rapport au PNB (1979 : 5,05\%; $1980: 2,69 \%$ ). Cela est dû essentiellement à l'importance des flux privés (investissements, crédits) gérés par les banques et les grandes entreprises du pays. Nul doute que cette prépondérance du secteur privé constitue une 
caractéristique importante du profil général des relations de la Suisse avec les pays en développement. Mais cette description resterait incomplète si I'on ignorait la portée de cet autre volet des apports "non-publics" que représentent les dons des organisations privées de coopération au développement.

Tout d'abord, en valeur absolue, les quelque cent millions de francs récoltés en 1981 par les organisations de coopération constituent près de $25 \%$ du total de I' "aide" (publique) suisse au développement et, si on rapporte cette somme à la population et que l'on compare ensuite le résultat avec celui qu'obtiennent d'autres pays, on mesure mieux l'importance de ce type de prestations :

\begin{tabular}{|c|c|c|c|c|c|c|}
\hline \multicolumn{7}{|c|}{1980} \\
\hline & $\begin{array}{c}\text { Total des dons } \\
\text { en millions de } \\
\text { US } \$\end{array}$ & Population & $\begin{array}{l}\text { PNB/habitant } \\
\text { en US \$ }\end{array}$ & don/habitant & $\begin{array}{c}\text { don/PNB } \\
\text { en } \%\end{array}$ & $\begin{array}{c}\text { dons/APD } \\
\text { en } \%\end{array}$ \\
\hline Allemagne & 420,7 & 61,3 & $13.453,5$ & $6,86 \$$ & 0.051 & 10,08 \\
\hline Canada & 102,0 & 23,5 & $10.485,1$ & $4,34 \$$ & 0.041 & 6,01 \\
\hline Etats-Unis & $1.301,0$ & 221,9 & $11.836,4$ & $5,86 \$$ & 0.049 & 15,77 \\
\hline France & 35,7 & 53,3 & $12.232,6$ & $0,66 \$$ & 0.005 & 0,75 \\
\hline Pays-Bas & 78,7 & 13,9 & $11.431,6$ & $5,66 \$$ & 0.049 & 4,89 \\
\hline Norvège & 33,0 & 4,1 & $13.975,6$ & $8,04 \$$ & 0.057 & 6,60 \\
\hline Suède & 59,0 & 8,3 & $14.650,6$ & $7,10 \$$ & 0.048 & 6,34 \\
\hline Suisse & 63,2 & 6,3 & $16.444,4$ & $10,03 \$$ & 0.061 & 24,49 \\
\hline
\end{tabular}

Sources

OCDE - Coopération pour le développement, Examen 1981, Paris, 1981, pp. 204-205, 257.

Banque mondiale, Rapport sur le développement dans le monde 1980, Washington DC, 1980, p.131.

Une telle statistique n'a qu'une valeur purement indicative, puisque, à l'évidence, tous les habitants ne contribuent pas aux collectes (1), que par ailleurs un tel tableau ne tient pas compte des disparités dues à l'inégalité du pouvoir d'achat des monnaies nationales $(2)$; il indique néanmoins une tendance, celle de l'engagement financier personnel d'une large fraction de la population suisse en faveur du "Tiers Monde". D'où le succès des organisations privées de coopération mises en place pour canaliser ces innombrables dons individuels. Mais ce foisonnement ne s'explique pas uniquement par l'existence d'une "tradition humanitaire" propre à notre pays : on veut bien donner, mais on veut savoir où va l'argent que l'on donne et l'on n'hésite guère à créer de nouvelles associations pour financer des projets spécifiques. Voilà pourquoi il est impossible de connaître exactement le nombre de ces 
organisations privées, ni le total réel des sommes qu'elles parviennent à recueillir. La statistique annuelle publiée par Swissaid recense près de nonante organismes récoltant ensemble plus de cent millions de francs, mais ces deux chiffres sont assurément faux car il existe une multitude de groupes (souvent d'orientation caritative ou centrée sur un projet particulier, mais aussi politique) ne figurant sur aucun registre et qui parviennent à mobiliser des fonds parfois importants pour les causes qu'ils soutiennent : ainsi, la statistique mentionne telle association qui a récolté 5.000 francs pour un projet en Colombie, mais elle omet telle autre qui a récolté plus de 300.000 francs en trois ans pour financer des projets au Cap Vert (3). Et que dire de tous les groupes informels (les habitants d'un même immeuble, les élèves d'une même classe, etc.) qui soutiennent directement un ami ou un parent missionnaire, lequel, grâce à ces dons, parvient à faire vivre une école, un poste sanitaire ou un modeste centre de formation agricole? On pourrait facilement multiplier les exemples qui donneraient sans doute la mesure de la générosité helvétique, mais qui montreraient au moins aussi clairement que la Suisse a la passion de l'argent bien géré, la hantise du gaspillage, et I'amour des comités de toutes sortes!

Présenter un tableau d'ensemble de ces organisations de coopération constitue une gageure. On peut toutefois, à grands traits, en donner un premier aperçu en retraçant leur évolution historique (4).

Ce sont, bien entendu, les sociétés missionnaires qui sont à l'origine de toutes les actions dites de "développement" car, dès leurs lointains débuts, elles ont toujours lié leur tâche d'évangélisation à des actions dites de "développement", avant même que la notion ait pris le sens qu'on lui connaît aujourd'hui. Un autre groupe d'organismes est formé par des institutions créées lors de la deuxième guerre mondiale (Entraide protestante aux Eglises et aux réfugiés : EPER), ou immédiatement à la fin du conflit, pour apporter une aide humanitaire aux populations européennes; progressivement, dès le milieu des années cinquante, elles se sont tournées vers les pays du "Tiers Monde" en complétant leurs activités caritatives traditionnelles par la mise sur pied de "projets de développement" (rappelons que l'Aide suisse à I'Europe fut fondée en 1947, qu'elle se transforma en 1956 en "Aide suisse à l'étranger", et qu'elle ne prit le nom de Swissaid qu'en 1968). Vers la fin des années cinquante se constituent les premières "organisations de coopération" au sens propre du terme (I'Aide suisse à des régions extra-européennes - devenue Helvetas en 1965 - fut créée en 1955, Swisscontact en 1959, sous le nom de Fondation suisse d'assistance au développement technique) qui excluent l'aide humanitaire de leurs buts et se consacrent entièrement à la pratique du "développement" par l'envoi d'experts, le financement de projets, la formation de cadres techniques et agricoles.

Ainsi, une infrastructure privée était déjà en place lorsque la Confédération créa, en 1961, le "Service du Délégué à la coopération technique", et c'est 
cette antériorité qui explique l'étroite collaboration qui s'instaura immédiatement entre les services gouvernementaux et les organisations de coopération, lesquelles obtinrent soit des subventions fédérales pour leurs propres projets, soit de gérer elles-mêmes (en régie) les projets officiels. Ce lien entre la coopération technique gouvernementale et les organismes privés autorisera ceux-ci à s'exprimer de plus en plus clairement dans les débats visant à déterminer une politique globale de la coopération.

Un quatrième groupe est constitué par les organisations, nées dès la fin des années soixante (Déclaration de Berne, Arbeitsgruppe Dritte Welt, Centre Europe-Tiers Monde (CETIM), etc.), qui ne financent aucun "projet de développement" mais cherchent d'abord à informer le public sur l'ensemble des relations économiques et politiques de la Suisse avec le "Tiers Monde". Jusqu'à cette période en effet, l'information diffusée par les organisations de développement concernait presque exclusivement les divers projets dont elles avaient la charge, et l'on manquait d'une vision plus large du rôle joué par notre pays - et singulièrement par les milieux économiques - dans les pays en développement. Tous ces groupes d'information, aux moyens d'action très différents, partageaient la conviction selon laquelle l'aide au développement ne devait pas tant consister à donner plus qu'à prendre moins. D'emblée, ils s'inscrivirent dans une perspective critique (qui était aussi celle de l'époque) tant vis-à-vis du gouvernement que des entreprises privées, voire même à l'égard des organisations "traditionnelles" soupçonnées d'être plus préoccupées du succès de leurs collectes que de la lutte contre les causes structurelles du sous-développement. Quant à leur tâche d'information, elle était relayée et renforcée par une multitude d'autres groupes, orientés de façon plus spécifique, qui s'étaient formés dans cette même période (Mouvement anti-apartheid de Suisse dès 1965, Comités Chili, Brésil,Vietnam, Uruguay, etc.).

Enfin, plus récemment encore, une dernière forme de coopération a vu le jour : elle cherche à transformer certaines pratiques commerciales soit en ouvrant le marché suisse à certains produits fabriqués dans le Tiers Monde tout en assurant aux producteurs une rémunération équitable et en informant le consommateur sur les conditions de production (OS3, Magasins du Monde), soit en faisant pression, par le biais des assemblées d'actionnaires, sur les grandes entreprises transnationales (Convention d'actionnaires Nestlé : CANES), soit encore en exprimant de façon tangible sa solidarité avec les grévistes d'entreprises du Tiers Monde (Solifonds).

Au terme de ce rapide survol, on voit bien comment cette évolution des organisations privées de coopération a accompagné celle de la "philosophie" dominante du développement : alors qu'il y a vingt ans chacun pensait que c'était "là-bas" qu'il fallait porter l'effort en priorité, on a petit à petit cherché à comprendre mieux les mécanismes du sous-développement pour se persuader enfin que le "développement" du Tiers Monde était lié à de profonds changements structurels dans les pays du "centre" et chez nous en 
particulier (5), et ce n'est pas un hasard si, parfois, ce furent les mêmes personnes qui participèrent à la fondation de mouvements de styles très différents pour répondre aux transformations du contexte international ou pour porter le combat sur de nouveaux fronts. Au fil des années, les positions des diverses organisations de coopération se sont certainement rapprochées : certes, les institutions qui financent des projets - et qui gèrent d'importants budgets - sont généralement plus "pragmatiques" dans leur approche politique que les organisations formées de militants, mais les disputes concernant les formes d'aide (humanitaire ou de projets) ont cessé et, depuis les années septante, l'information dispensée par la quasi-totalité des organisations est devenue plus engagée, surtout si l'on tient compte du fait que certaines grandes organisations de coopération "traditionnelles" ont parfois créé leur propre "centrale d'information" indépendante (I3H) ou qu'elles contribuent au financement de certains groupes plus militants; enfin, certaines organisations, naguère orientées uniquement sur l'information, entreprennent elles aussi des actions concrètes (vente de sacs de jute en provenance du Bengladesh, boycott des ananas des Philippines, etc.) qui constituent le "support" d'une prise de conscience.

Cela dit, l'importance des organisations privées ne tient pas seulement à leur nombre, aux sommes qu'elles parviennent à récolter ou à l'audience à laquelle elles peuvent prétendre, mais aussi à leur participation à l'élaboration de la doctrine officielle sur le développement. Sans doute cela conditionnet-il ceci : la part considérable qu'elles prennent dans la formation de l'opinion publique oblige le pouvoir politique à les prendre au sérieux. Mais ce qui apparaît aujourd'hui comme une évidence aurait pu évoluer de façon bien différente : le fait qu'une bonne partie des organismes qui gèrent des "projets de développement" reçoivent aussi des contributions fédérales aurait pu transformer ceux-ci en simples "courroies de transmission" de la vision officielle du "développement". Or, on peut dire que ce ne fut jamais le cas, même au cours des années soixante, puisqu'alors la doctrine officielle apparaissait plutôt comme le reflet de celle des organisations privées...

Cinq événements différents peuvent servir à caractériser l'évolution des rapports des organisations privées et du Service de la coopération technique (DDA).

En 1970, les Eglises (auxquelles sont liées un bon nombre d'organisations de coopération) convoquèrent à Berne une "conférence interconfessionnelle Suisse - Tiers Monde" et y invitèrent, outre les représentants des organisations privées "traditionnelles", ceux du gouvernement, de l'industrie privée, des partis politiques ainsi que des personnes du Tiers Monde. Le débat, sans cesse relancé par les délégués des nouveaux groupes concernés d'abord par l'information, fut surtout critique à l'égard de l'économie privée et de la Division du commerce, et relativement favorable aux efforts du Service de la coopération technique; même si I'on commençait à remettre en cause les rapports structurels entre le "centre" et la "périphérie", on n'en soutenait 
pas moins les initiatives privées dans le cadre de l'aide publique au développement, et l'on recommandait un accroissement des engagements financiers de la Confédération (6).

Cinq ans plus tard, la situation était toute différente; dans le cadre de la procédure de consultation, on discutait depuis 1972 sur le projet de loi sur la coopération au développement et l'aide humanitaire. Le Parlement avait cherché à gagner du temps et à éviter un référendum en demandant au gouvernement un rapport complémentaire, à la suite de la flambée des prix pétroliers, mais il ne parvenait toujours pas à se mettre d'accord sur un texte final. La divergence fondamentale résidait dans l'article 5 de la loi dans lequel les organisations privées tenaient à stipuler que l'aide publique devait essentiellement s'adresser "aux groupes de population les plus défavorisés", et cela contre l'avis du gouvernement et des partis de droite qui estimaient la précision inutile. Pour faire connaître leur position, les organisations privées donnèrent mandat à une vingtaine d'experts de rédiger des "propositions pour une nouvelle politique suisse de développement"; celles-ci parurent en allemand en mars 1975 et en français en juin de la même année sous le titre "Maldéveloppement Suisse-Monde" (7). Ce rapport - accepté par toutes les organisations mandataires, à l'exception de Swisscontact et de Caritas - se rattachait très clairement à la "théorie de la dépendance", montrait que le monde industrialisé comme le Tiers Monde souffraient l'un et l'autre de "mal-développement" et réclamait d'importants changements de structures tant au "nord" qu'au "sud". Ce texte fut très fraîchement accueilli par le conseiller fédéral Graber, mais il fit très rapidement l'objet de nombreux débats à l'intérieur du Service de la coopération technique, où de nombreux fonctionnaires sympathisaient avec les thèses de la commission des organisations privées, sans toutefois les adopter entièrement. Finalement, la loi sur le développement fut adoptée en automne 1975; son article 5 était conforme aux vœux des organisations privées! Cet exemple montre clairement l'importance de celles-ci dans le débat politique, et le poids qu'elles représentent dans la définition de la doctrine officielle (8).

On en eut un autre exemple en 1976, lors de la votation populaire concernant un prêt de deux cent millions de francs à I'IDA. Celui-ci avait été d'abord combattu au Parlement par les Républicains de M. Schwarzenbach qui lancèrent un référendum contre cette forme d'aide multilatérale. La plupart des partis politiques, le gouvernement et les milieux économiques faisaient campagne pour le "oui" et cette belle unanimité semblait assurer leur victoire. Mais, à la suite du rapport "Maldéveloppement Suisse-Monde", les organisations privées ne pouvaient pas, sans autre, appuyer une mesure dont I'application risquait de ne pas profiter "aux groupes de population les plus défavorisés". Certaines d'entre elles recommandèrent un "oui" plutôt timide, assorti de conditions et de réserves qui semèrent le doute dans l'esprit de nombreux citoyens. D'autres, comme la Déclaration de Berne, conseillèrent à leurs adhérents de voter blanc (pour se démarquer à la fois 
du gouvernement et des Républicains), au grand regret du Service de la coopération technique qui n'avait pas ménagé ses efforts pour rallier des voix à sa cause. Au vote, le prêt de I'IDA fut refusé par 56,5\% des voix "grâce" ou "à cause" de la conjonction de l'opposition "de droite" et "de gauche", et l'on peut dire que les organisations privées de coopération jouèrent en l'occurrence le rôle d'arbitres dans un scrutin beaucoup plus serré que prévu.

On aurait tort de croire, à partir de ces deux cas, que les relations entre les organisations privées et la DDA ont évolué de façon divergente. Certes, les organisations "militantes" restent critiques à l'égard de la politique officielle, mais celle-ci n'est pas le fait exclusif de la DDA, tant s'en faut! Qu'un coup d'Etat dans tel pays du Tiers Monde ne soit pas assez clairement condamné, c'est le Département des affaires étrangères qui est attaqué; que les crédits d'aide publique soient rognés pour des raisons conjoncturelles, c'est au Conseil fédéral et au Parlement que I'on s'en prend; que la politique d'asile soit trop restrictive à l'égard de certains pays, c'est le Département de justice et police que I'on critique; que des crédits mixtes soient accordés à certains régimes musclés, c'est d'abord à l'OFAEE que I'on s'en prend. Finalement, personne n'est épargné, pas même les Eglises, ni les grandes organisations de coopération. On le vit clairement lors du Symposium de la solidarité qui se tint à Berne, en 1981, et auquel.participèrent près de quarante organisations militantes sous la devise : “le développement, c'est la libération" (9). Certes, les grandes ONG s'abstinrent de soutenir cette manifestation, mais cela ne les empêche pas de passer au crible - même si elles le font parfois avec une certaine discrétion - la politique globale de la Confédération, surtout lorsqu'elle se trouve en contradiction avec le principe de solidarité considéré souvent comme le corollaire de notre neutralité. En revanche, c'est avec les milieux de l'économie privée que les relations sont de plus en plus tendues, d'autant plus que ceux-ci, pour faire face à la récession, doivent pratiquer à l'égard du Tiers Monde une politique commerciale toujours plus agressive qui est souvent loin de tenir compte des intérêts des groupes de population les plus défavorisés. L'histoire récente de la "nouvelle organisation" (N.O.) le montre bien (10). En I'occurrence, ce sont les organisations de coopération qui ont obtenu gain de cause puisque I'organisme a été effectivement créé sous le nom d'Intercoopération; pour des raisons juridiques, la DDA n'en fait pas partie, mais les entreprises privées ne sont pas parvenues non plus à s'y immiscer : le projet initial, renforçant la collaboration entre les organisations privées et le gouvernement, a été réalisé malgré les oppositions qu'il a rencontrées.

Pour toutes ces raisons, il faut bien convenir que les organisations privées constituent un des acteurs majeurs de la politique de développement en Suisse. Bien entendu, on ne peut leur attribuer un contenu politique univoque, même si, en leur sein, les principales thèses proposées par le rapport "Maldéveloppement Suisse-Monde" sont aujourd'hui largement acceptées. 
On peut toutefois résumer à grands traits leur évolution en disant qu'elles furent, à leur origine, l'expression d'une certaine bonne conscience (caractérisée par l'assurance de détenir un certain nombre de valeurs, de connaissances techniques et de moyens dont le transfert dans le Tiers Monde allait mettre fin au sous-développement) et qu'elles sont devenues, au fil des années, le révélateur de la mauvaise conscience d'un pays qui, installé dans sa richesse, son ordre et son confort, assiste, impuissant, aux drames que vivent les autres et que les mass-media lui présentent quotidiennement. Alors que certains prennent prétexte de la politique de neutralité pour justifier leur indifférence, les organisations privées s'efforcent au contraire de pratiquer une solidarité active avec les plus défavorisés et de dénoncer avec la plus grande énergie toutes les complicités repérables entre les classes dirigeantes des pays du Tiers Monde et celles de la Suisse. On risque de n'y voir qu'une volonté de moraliser la vie publique (ou, ce qui revient au même, de n'y trouver que le reflet d'une "idéologie" sans lien avec la "réalité") (11). Et s'il ne s'agissait, finalement, que de l'application concrète de ce vieux slogan de l'aide au développement: "nous sommes tous dans le même bateau"? Car le maintien de nos libertés passe aussi par la libération des exploités.

\section{NOTES}

1. Dans son enquête, parue en 1971 (Schweizer und Entwicklungshilfe, Inneansichten der Aussenpolitik,Reihe Res publica, Paul Haupt, Bern/Stuttgart, 2 Bände), Gerhard Schmidtchen montre toutefois que seul $36 \%$ de la population âgée de plus de seize ans n'a jamais contribué à aucune collecte au cours des deux dernières années considérées.

2. II faut rappeler aussi - pour briser tout chauvinisme - que la Suisse est le pays le plus riche du monde (après le Koweit, mais avant les autres pays industrialisés) si I'on calcuie le PNB/hab.

3. Nous pensons ici à l'Association Cap-Vert Genève : bien qu'ayant commencé son travail en 1979, elle ne figurait pas, jusqu'ici, sur la statistique annuelle de Swissaid (intitulée Aide suisse aux pays en développement). II faut, pour être juste, préciser que l'Association récolte une part très importante de ses fonds auprès des communes et du canton de Genève : ceux-ci sont donc recensés dans la statistique, mais les dons du public n'y apparaissent pas.

4. On trouvera des informations plus détaillées sur l'évolution de la "doctrine" de la coopération dans l'ouvrage de Jean-Jacques de Dardel - La coopération au développement, certitudes et interrogations, collection Etudes de développement, IUED, Genève, 1981.

5. Sur les transformations du style de l'information des organisations de coopération, cf. Gilbert Rist - Image des autres, image de soi? Comment les Suisses voient le Tiers Monde, Collection Etudes de Développement, Georgi, Saint-Saphorin, 1978.

6. L'initiative de cette conférence avait été prise, en fait, par la Commission Tiers Monde de l'Eglise nationale protestante de Genève, en 1968. Cette Commission était présidée alors par Pierre Bungener, directeur de I'Institut africain (aujourd'hui IUED) et elle comptait parmi ses membres le professeur André Biéler, théologien et 
économiste, qui fut à l'origine de nombreux mouvements de conscientisation, en Suisse, sur les problèmes du Tiers Monde. Quant au compte rendu final de la Conférence interconfessionnelle de 1971, il a été publié par Hans K. Schmocker et Michael Traber (éditeurs) sous le titre Berichte und Dokumente der Interkonfessionnellen Konferenz in Bern, TVZ Verlag, Zürich / Imba Verlag, Freiburg, 1971.

7. Entwicklungs/and Welt - Entwicklungsland Schweiz, Vorschläge für eine neue schweizerische Entwicklungspolitik, Z-Verlag, Basel, 1975; Maldéveloppement Suisse-Monde, Propositions pour une nouvelle politique suisse de développement, CETIM, Genève, 1975.

8. Un aperçu des débats qui eurent lieu autour de ce petit libelle a été publié par Peter Braunschweig et Bruno Gurtner (éditeurs) sous le titre : Zur Diskussion gestel/t : Entwicklungs/and Welt - Entwicklungs/and Schweiz, Z-Verlag, Basel, 1976.

9. On consultera à ce sujet le Livre noir Suisse-Tiers Monde, Quelques exemples concrets, "Que faire ?", Genève, 1981; Schwarzbuch Schweiz-Dritte Welt, Fallbeispiele, 1981.

10. Cf. Jacques Forster, "La nouvelle contestation de la coopération au développement", Annuaire Suisse-Tiers Monde 1981, No 1, IUED, Genève, pp. 213-222 et ibid. pp. 92-95.

11. Cf. I'article de Robert Jenny dans le présent Annuaire, "Eine Entideologisierung (...) des Entwicklungszusammenarbeit erscheint uns vordringlich". 